\title{
Management of Maxillary Mucormycosis - A Case Report in Bangladesh
}

\author{
Rahman $\mathrm{QB}^{1}$, Rahaman $\mathrm{MM}^{2}$, Imon $\mathrm{AA}^{3}$, Rabby $\mathrm{MAI}^{4}$, Chowdhury MAJ ${ }^{5}$
}

\begin{abstract}
Mucormycosis refers to several different disease caused by infection in the order of mucorales. Rhizopus species are the most common causative organism. Most mucormycosis infections are life-threatening and risk factor such as diabetic ketoacidosis and neutropenia, are present in most cases. Severe infection of the facial sinuses, which may extend into the brain, is the most common presentation. We describe our clinical experience with a case of mucormycosis of the maxilla. Early diagnosis and prompt treatment can significantly reduce the mortality and morbidity of this lethal fungal infection.
\end{abstract}

\section{Introduction}

Mucormycosis is the common name given to several different diseases caused by fungi of the mucorales ${ }^{1}$. The patient generally has uncontrolled diabetes mellitus and is acidotic, has a hematological malignant disease like leukemia, or is receiving immunosuppressive therapy ${ }^{2,3}$. Usually mucormycosis presents as an acute infection and manifests in rhinocerebral, pulmonary, gastrointestinal, cutaneous, or disseminated forms, ${ }^{4}$ rarely affecting otherwise healthy people 5 . The infection begins in the upper turbinate or paranasal sinuses ${ }^{6,7}$ or less commonly in the palate or pharynx. The most common presentation in the head and neck region is maxillary and orbital cellulitis in a person with inadequately controlled diabetes mellitus ${ }^{7}$.

1. Prof. Quazi Billur Rahman

Professor \& Chairman of Department of OMS

Bangabandhu Sheikh Mujib Medical University, Dhaka

2. Corresponding Author:

Dr. Muhammad Mizanur Rahaman, BDS, FCPS

Maxillofacial Surgeon

E-mail: mizanddc@yahoo.com

3. Dr. Ashik Abdullah Imon, BDS, MS (Phase-B, OMS) Bangabandhu Sheikh Mujib Medical University, Dhaka

4. Dr. Md. Atiqul Islam Rabby, BDS

Dental Surgeon

Pacific Dental Care, Farmgate, Dhaka

5. Prof. M.A Jalil Chowdhury

Professor of Medicine

Bangabandhu Sheikh Mujib Medical University, Dhaka
Since mucormycosis occurs infrequently, it may pose a diagnostic and therapeutic dilemma for those who are not familiar with its clinical presentations.

\section{Case report}

A 68 years old male patient reported to us with exposed bone in the upper jaw for 2 months. The patient medical history showed diabetes mellitus for 20 years. He had been on oral hypoglycemic treatment for first 10 years and on insulin for 10 years. He had a history of wearing removable partial denture due to missing upper anterior teeth for 2 years. Last 2 months he noticed a small area of gray coloured exposed bone in the maxillary anterior alveolar region under the denture. He took some medicine and his doctor done local debridement and extraction of other broken teeth and retained root but the wound \& extraction socket was not healed. On extra oral examination there was mild swelling in the whole upper jaw. An intra-oral examination revealed exposed nectoric gray colored bone on both maxilla involving anterior \& posterior alveolar region \& mid palatal area. A computed tomography (CT) scan showed mucosal thickening with lobulated soft tissue density area within the both maxillary sinuses along with destruction of the walls of maxillary sinus, hard palate. Extension also seen in the right nasal cavity. Erosion of the nasal bones and frontal processes of maxilla is seen at both sides. No definite intra-orbital extension of these lesion are seen.

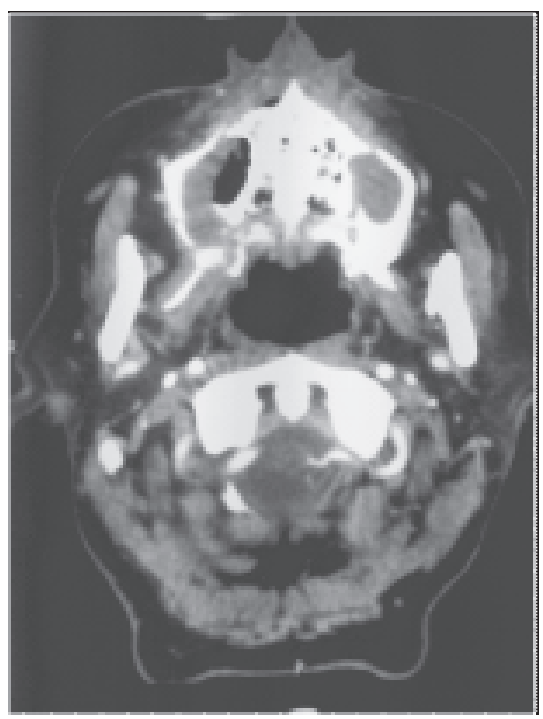

Figure 1: Pre operative CT scan of the patient 


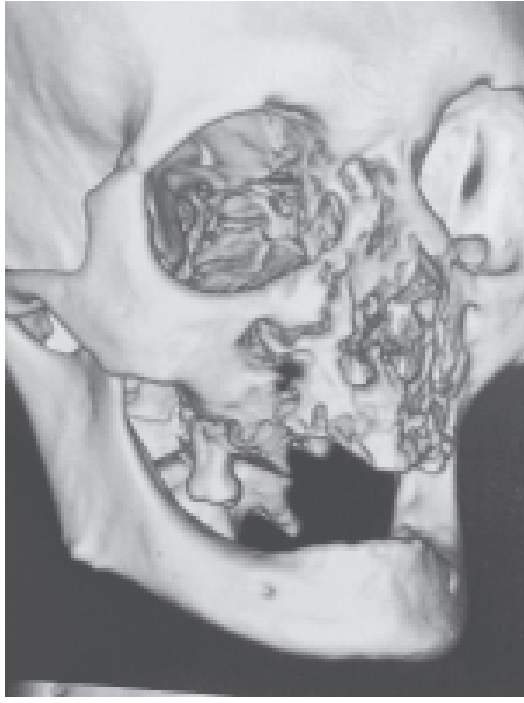

Fig 2: 3D reconstruction of CT scan.

A provisional diagnosis of maxillary osteomyelitis was made. Biopsy of hard tissue along with adjacent soft tissue was taken under local anesthesia and sent for microbiological and histopathological examination. Microbiological examination revealed broad, aseptate , ribbon like hyphae, and this was hematoxylin and eosin (H\&E) stain readily identified non-septate mucormycotis hyphae. Following confirmation of the diagnosis, the patient hospitalized and his blood sugar was controlled by the physician. During this period voriconazole $400 \mathrm{mg}$ orally was given twice daily for 4 days. After Operation 4 days voriconazole twice daily \& 15 days injection amphotericin B once daily was given. On surgical exploration the necrotic bone piece was found to be loose and was removed, leading to opening of the antrum. The antral lining was found to be thick and fibrous. Maxillary sequestrectomy, total antral curettage and complete debridement were carried out. Aggressive surgical resection was done by taking $1 \mathrm{~cm}$ free margin. Maxillary obturator was given \& stitched with the wound margin.

Post operative histopathology report was mucormycosis . Intravenous amphotericin B was considered in the post operative phase and the patient was monitored for nephrotoxicity. Follow up of the patient was satisfactory.

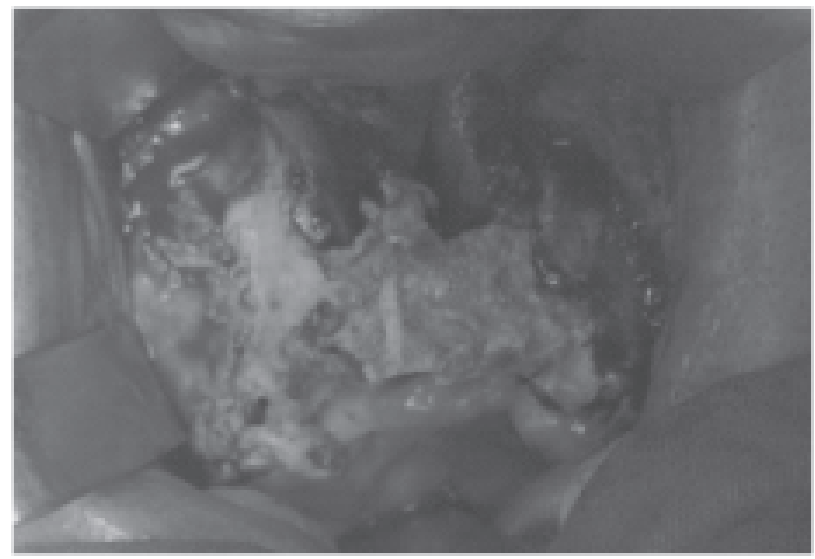

Fig 3: Exposed bone with infected margin.

MEDICINE 2015 Volume 27 Number 01

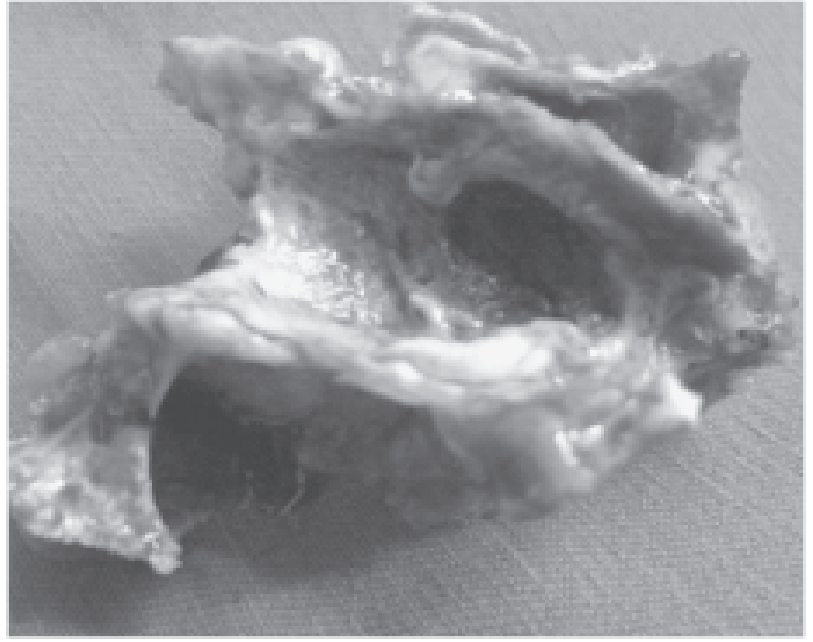

Fig 4: After excision infected and necrosed part of maxilla

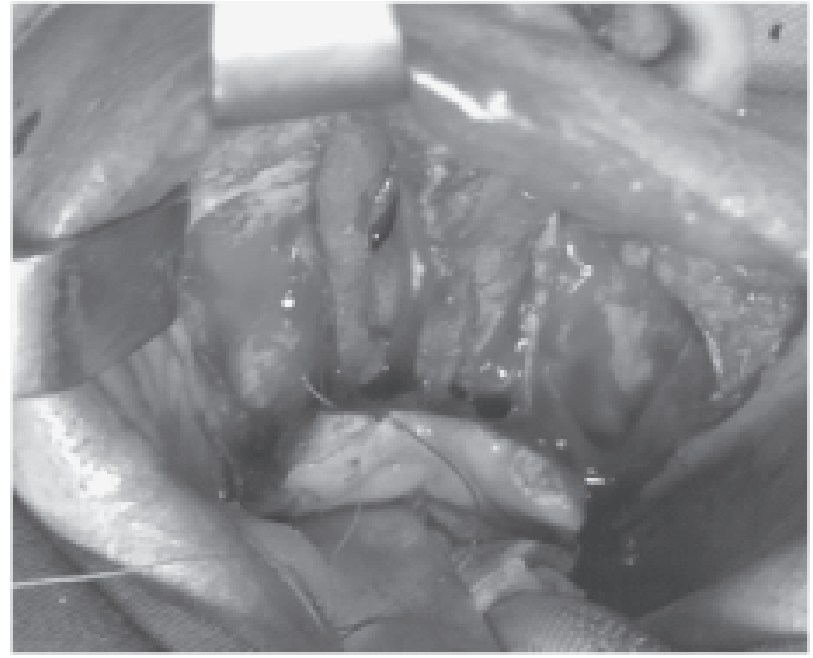

Fig 5: After excision of necrosed tissue antrum exposed

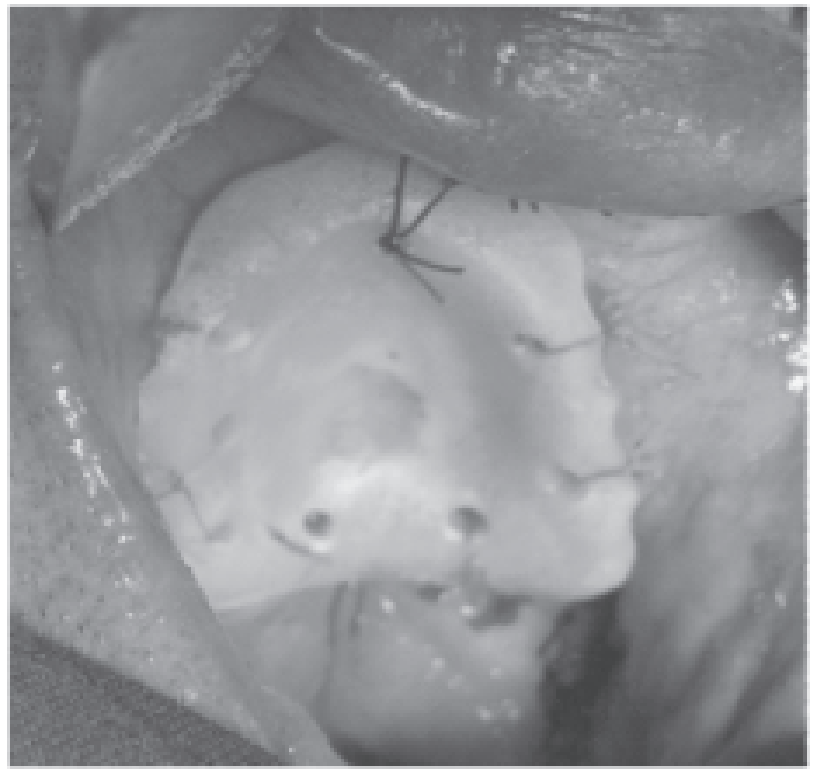

Fig 6: Maxillary obturator was stitched with the wound margin. 


\section{Discussion}

In routine maxillofacial practice, intra-oral exposed bone (maxillary necrosis) is generally diagnosed as osteomyelitis. Maxillary necrosis can occur due to bacterial osteomyelitis, herpes zoster, trauma, iatrogenic infections, or fungal infections, such as mucormycosis, aspergillosis, etc. Mucormycosis is a life-threatening disease and medical management alone is not effective because of poor drug delivery to the infection site due to extensive vascular thrombosis. ${ }^{8}$ the fungus invades the arteries leading to thrombosis that subsequent causes necrosis of hard \& soft tissue. The ultimate outcome of mucormycosis depends in large part on the prognosis of the underlying disease and the ability to reverse the predisposing condition. ${ }^{1}$ The overall mortality rate has been shown to be $50 \%$, although higher survival rates up to $80-85 \%$ have recently been reported..$^{9-11}$ The associated prognostic factor depends on the extent of disease, including orbital or intracranial extension in cases of rhinocerebral mucormycosis. Rhinocerebral mucormycosis is one of the most rapidly progressing and lethal forms of fungal infection in humans and usually begins in the nose and paranasal sinuses. ${ }^{4}$ In diabetic patients there is a high incidence of mucormycosis caused by Rhizopus arrhizus, because they produce the enzyme ketoreductase, which allows them to utilize the patient's ketone bodies. ${ }^{7}$ Aggressive correction of hyperglycemia and acidemia, followed by aggressive resection-type debridement is needed. The general approach is to diagnose early and to treat early and aggressively with all modalities available.

The current mortality is not high due to better diagnosis and management of the usual comorbidities. As shown in various studies, the control of underlying predisposing illness and aggressive surgical debridement, along with prompt parenteral administration of amphotericin $B$ remains the essential treatment today. ${ }^{12-14}$

Though amphotericin B formulations have remained the mainstay of treatment for mucormycosis, recent studies have demonstrated that posaconazole, an extended-spectrum triazole is currently considered a second-line drug for treatment of mucomycosis and the typical dose is $400 \mathrm{mg}$ twice daily (total $800 \mathrm{mg} / \mathrm{d}$ ). Administration with a high-fat meal/food and acidic beverage enhances absorption of the drug. Patient on pasconazole should avoid antacid, especially proton pump inhibitors.

A diabetic patient with exposed necrotic bone with a history of tooth extraction or wearing a denture should alert the clinician to a possible mucormycotic infection. Prompt and aggressive treatment is required to ensure a favorable outcome.

\section{References}

1. Sugar AM. Agents of mucormycosis is and related species. In: Mandell, Bennett, Dolin, editors. sixth ed., Mandell Douglas and Bennett's principles and practice of infectious diseases, Vol. 2, sixth ed. Philadelphia, Pennsylvania USA: Elsevier Churchill Livingstone; 2005 .p. 2973-84.

2. Muresan A. A case of cerebral mucormycosis diagnosed in life with eventual recovery. J Clin Pathol 1960; 13:34-6.

3. Rosen PP. Opportunistic fungal infections in patients with neoplastic disease. Pathol Ann 1976;11:255-315.

4. Leitner C, Hoffmann J, Zerfowski M, Reinert S. Mucormycosis: necrotizing soft tissue lesion of the face. J Oral Maxillofac Surg 2003;61:1354-8.

5. Singh J, Prasdnna NM. Phycomycosis in an apparently normal host. J Otolaryngol 1977;6:37-42.

6. Abidi E, Sismanis KC, Pastore P. Twenty-five years experience treating cerebrorhino orbital mucormycosis. Laryngoscope 1984;94:1060-2.

7. Morduchowicz G, Schmueli D. Rhinocerebral mucormycosis in renal transplant patients: report of 3 cases and review of literature. Rev Infect Dis 1986; $8: 444-$

8. Smith JL, Stevens DA. Survival in cerebro-rhino-orbital zygomycosis and cavernous sinus thrombosis with combined therapy. South Med J 1986;79:501-4.

9. Parfrey NA. Improved diagnosis and prognosis of mucormycosis: a clinicopathological study of 33 cases. Medicine (Baltimore) 1986;65:113-23.

10. Salisburg PL, Caloss R, Cruz JM, Powell BL, Cole R, Kohut RI. Mucormycosis of the mandible after dental extractions in a patient with acute myelogenous leukaemia. Oral Surg Oral Med Oral Pathol Oral Radiol Endod 1997;83:340-4.

11. Auluck A. Maxillary necrosis by mucormycosis: a case report and literature review. Med Oral Pathol Oral ir Bucal 2007;12:360-4.

12. Jung SH, Kim SW, Park CS, Song CE, Cho JH, Lee JH, et al. Rhinocerebral mucormycosis: consideration of prognostic factors and treatment modality.Int J Oral HNS 2009;36:274-9.

13. Kemer J, Kuijper EJ, Mirck PG, Balm AJ. Recovery from rhinocerebral mucormycosis in a ketoacidotic diabetic patient: a case report. J Laryngol Otol 1993;107:233-5.

14. Dhiwakar M, Thakar A, Bahadur S. Improving outcomes in rhinocerebral mucormycosis: early diagnostic pointers and prognostic factors. J Laryngol Otol 2003;117:861-5. 